Series A

I. MATHEMATICA

422

\title{
THE NON-PLESSNER POINTS \\ FOR THE SCHWARZ TRIANGLE FUNCTIONS
}

BY

PAUL GAUTHIER

H E L S I N I 1968

SUOM A L A I E N T I E D E A K T T M I A

doi:10.5186/aasfm.1969.422 
Communicated 9 February 1968 by P. J. Myrberg and K. I. Virtanen 


\section{The non-Plessner points for the Schwarz triangle functions*}

\section{Introduction}

Let $\mu(z)$ be the elliptic modular function defined in the unit disc $D:|z|<1$. It has been shown by Bagemihl [1, theorem 1] that the set of Plessner points [6, p. 59] for $\mu(z)$ is of measure $2 \pi$ and residual in the unit circle $C:|z|=1$. On the other hand $\mu(z)$ has angular limits at each of countably many parabolic vertices for $\mu(z)$, and so these points are certainly not Plessner points. It is natural to ask whether these are the only non-Plessner points. In this note it is shown that there are other nonPlessner points for $\mu(z)$. Indeed it is shown that the set of non-Plessner points for $\mu(z)$ has the cardinality of the real line.

Some of the results contained in this paper formed part of the author's doctoral dissertation presented to Wayne State University in 1967. The author wishes to thank Professor W. Seidel who directed the research, and both the University of Massachusetts and National Science Foundation who supported the research.

\section{Preliminary Results}

For any two points $z^{\prime}, z \in D$, we denote by $\varrho\left(z^{\prime}, z\right)$ the non-Euclidean hyperbolic distance between $z^{\prime}$ and $z$; for any two points $w$ and $w^{\prime}$ of the Riemann sphere, we denote by $\chi\left(w, w^{\prime}\right)$ the chordal distance between $w$ and $w^{\prime}$. For a set $S \subset D$ and $r>0$, we define

$$
H(S, r)=\{z: \varrho(S, z) \leq r\} .
$$

The cluster set $C(f, S)$ of $f(z)$ on $S$ is the set of all values $w$ of the Riemann sphere such that there is a sequence $\left\{z_{n}\right\}, z_{n} \in S$, such that $\left|z_{n}\right| \rightarrow 1$ and $f\left(z_{n}\right) \rightarrow w$. For $S$ a Stolz angle or a segment in $D$ with only one end point on $C$, Rung [7] has introduced the notation

$$
\hat{C}(f, s)=\bigcap_{\Delta} C(f, \Delta),
$$

*) Presented at the meeting of the American Mathematical Society, University of Chicago, Chicago, U.S.A., April 15, 1967. 
where $\Delta$ varies over all Stolz angles which properly contain $S$. We note that

$$
\hat{C}(f, S)=\bigcap_{r>0} C(f, H(S, r)) .
$$

For this reason we now define $\hat{C}(f, S)$ by (1) for any set $S \subset D$. A subset $S \subset D$ is called a $\varrho$-set if there is a sequence $\left\{z_{n}\right\}$ of $\varrho$-points [3] for $f(z)$ with $z_{n} \in S, n=1,2, \ldots$

Theorem 1. Let $w=f(z)$ be a function meromorphic in $D$ and let $S \subset D$. Then either $S$ is a $\varrho$-set or

$$
C(f, S)=\hat{C}(f, S) .
$$

Proof. If the closure of $S$ does not intersect $C$, then

$$
C(f, S)=\hat{C}(f, S)=\varnothing,
$$

and so there is nothing to prove.

Suppose on the other hand that the closure of $S$ meets $C$ and that

$$
C(f, S) \neq \hat{C}(f, S)
$$

Then since

$$
C(f, S) \subset \hat{C}(f, S)
$$

there must be a point $w_{0} \in \hat{C}(f, S)$ for which $w_{0} \notin C(f, S)$. For each positive integer $n, w_{0} \in C(f, H(S, 1 / n))$, and so one can find a point $z_{n}$ in $H(S, 1 / n)$ such that $\left|z_{n}\right|>1-1 / n$, and

$$
\chi\left(f\left(z_{n}\right), w_{0}\right)<1 / n .
$$

Since $z_{n}$ is in $H(S, 1 / n)$, there is a point $z_{n}^{\prime}$ in $S$ such that $\varrho\left(z_{n}, z_{n}^{\prime}\right) \leq 1 / n$. For large $n$, the chordal distance between $f\left(z_{n}^{\prime}\right)$ and $w_{0}$ must be bounded away from zero since $w_{0} \notin C(f, S)$. Hence by (3) we have that

$$
\varrho\left(z_{n}, z_{n}^{\prime}\right) \rightarrow 0 \text {, and } \chi\left(f\left(z_{n}\right), f\left(z_{n}^{\prime}\right)\right)>R, \text { for } n=1,2, \ldots,
$$

where $R$ is some fixed positive number. It follows [3] from (4) that $\left\{z_{n}^{\prime}\right\}$ is a sequence of $\varrho$-points, and so $S$ is a $\varrho$-set. This concludes the proof.

Corollary 1. Let $w=f(z)$ be a normal meromorphic [6, p. 86] function in $D$, and let $S \subset D$. Then

$$
\hat{C}(f, S)=C(f, S)
$$

This generalizes a result due to Rung [7]. 
Proof. By modifying a result of Gavrilov [4], we have shown [3] that a normal meromorphic function cannot possess a sequence of $\varrho$-points. Hence (5) follows from theorem 1.

We shall call two sets, $S_{1} \subset D$ and $S_{2} \subset D$, equivalent if for each $r>0$, there is a $\delta>0$ such that

$$
\begin{aligned}
& S_{2} \cap\{z:|z|>1-\delta\} \subset H\left(S_{1}, r\right) \\
& S_{1} \cap\{z:|z|>1-\delta\} \subset H\left(S_{2}, r\right) .
\end{aligned}
$$

and

This definition obviously has the following corollary.

Corollary 2. Let $w=f(z)$ be a meromorphic function in $D$, and let $S_{1}$ and $S_{2}$ be equivalent subsets of $D$. Then

$$
\hat{C}\left(f, S_{1}\right)=\hat{C}\left(f, S_{2}\right)
$$

Theorem 2. Let $w=f(z)$ be a normal meromorphic function in $l$ ). and let $S_{1}$ and $S_{2}$ be equivalent subsets of $D$. Then

$$
C\left(f, S_{1}\right)=C\left(f, S_{2}\right)
$$

Proof. This theorem follows directly from corollary 1 and corollary 2.

\section{Triangle functions}

Lemma 1. Any Schwarz triangle function in $D$ is normal.

Proof. Suppose $f(z)$ is a Schwarz triangle function (see [2]) defined in $D$, and suppose that $\left\{z_{n}\right\}$ is a sequence of $\varrho$-points for $f(z)$. For each $z_{n}$, let $\zeta_{n}$ be a point of the fundamental polygon for $f(z)$ such that $z_{n}$ is equivalent to $\zeta_{n}$. Then $\left\{\zeta_{n}\right\}$ is also a sequence of $\varrho$-points. Let $\zeta_{0}$ be a limit point of $\left\{\zeta_{n}\right\}$, and let $\left\{\zeta_{n(k)}\right\}$ be a subsequence of $\left\{\zeta_{n}\right\}$ which converges to $\zeta_{0}$. $\left\{\zeta_{n(k)}\right\}$ is also a sequence of $\varrho$-points, and so $f(z)$ assumes each value of the Riemann sphere, with at most two exceptions, infinitely often in every neighborhood of $\zeta_{0}$. Hence $\zeta_{0}$ lies on the unit circle. But $\zeta_{0}$ is a boundary point of the fundamental polygon, and so $\zeta_{0}$ is a parabolic vertex. It follows that $f(z)$ has an angular limit at $\zeta_{0}$. The sequence $\left\{\zeta_{n(k)}\right\}$ lies in the fundamental polygon and so it must approach $\zeta_{0}$ nontangentially. Hence $f(z)$ assumes every value of the Riemann sphere, with at most two exceptions, infinitely often in some Stolz angle having vertex at $\zeta_{0}$. This contradicts the fact that $f(z)$ has an angular limit at $\zeta_{0}$. Thus the supposition that $f(z)$ has a sequence of $\varrho$-points was false. By a theorem of Gavrilov (see [3]), then, $f(z)$ must be normal.

Theorem 3. The set of non-Plessner points for any Schwarz triangle function $f(z)$ has the cardinality of the real line. 
Proof. Let $\left\{T_{n}\right\}$ be the group of linear transformations with respect to which $f(z)$ is an automorphic function. For any $S \subset D$ we write $S_{n}$ for $T_{n}(S)$. We now define a subset $\bar{M}_{0}$ of the unit circle. We shall say that $\alpha \in \bar{M}_{0}$ if there is some non-Euclidean straight line $L$ which intersects the unit circle at $\alpha$ and for which the set $\cup\left\{L_{n}: n=1,2, \ldots\right\}$ is not dense in $D$.

Let $\alpha \in \bar{M}_{0}$ and let $L$ be as in the above paragraph. Since $\cup\left\{L_{n}: n=\right.$ $1,2, \ldots\}$ is not dense in $D$, there must be an open set $G$ contained in a fundamental region for $f(z)$ such that $\cup\left\{L_{n}: n=1,2, \ldots\right\}$ does not meet $G$. Hence $f(G)$ does not meet $f(L)$. Since $f(G)$ is open, $f(L)$ cannot be dense in the Riemann sphere, and so $C(f, L)$ is not total. Let $\beta$ be the other (besides $\alpha$ ) point at which $L$ intersects the unit circle, and let $S=L \cap\{z:|z-\alpha|<|z-\beta|\}$. Let $R$ be the radius $\arg z=\arg \alpha$. Then clearly $R$ is equivalent to $S$ and so by theorem 2,

$$
C(f, R)=C(f, S) .
$$

Since $S \subset L$ and $C(f, L)$ is not total, it follows from (8) that $C(f, R)$ is not total. According to a theorem of Bagemihl [1, lemma], it follows that the point $\alpha$ is not a Plessner point for $f(z)$. Hence no point of $\bar{M}_{0}$ is a Plessner point. Now Myrberg [5, p. 408] has shown that the set $\bar{M}_{0}$ has the cardinality of the real line, and so the proof is complete.

\author{
Wayne State University \\ Detroit, Michigan, U.S.A.
}

\title{
Bibliography
}

[1] Bageminl, F., Some approximation theorems for normal functions. - Ann. Acad. Sci. Fenn. A I 335 (1963), 1-5.

[2] Carathéodory, C., Funktionentheorie, Vol. 2. - Basel, 1950.

[3] Gauthier, P., A criterion for normalcy (submitted to Nagoya Math. J.).

[4] Gavrilov, V. I., On the distribution of values of non-normal meromorphic functions in the unit disc (Russian). - Mat. Sb. 109 (n.s. 67) (1965), 408-427.

[5] Myrberg, P. J., Ein Approximationssatz für die fuchsschen Gruppen. - Acta. Math. 57 (1931), 389-409.

[6] Noshiro, K., Cluster sets. - Berlin, 1960.

[7] Rung, D. C., Boundary behavior of normal functions defined in the unit disc. Mich. Math. J. 10 (1963), 43-51. 